\title{
Transient LES of an offshore wind turbine
}

\author{
Lukas Vollmer $^{1,2}$, Gerald Steinfeld ${ }^{1}$, and Martin Kühn ${ }^{1}$ \\ ${ }^{1}$ ForWind, Carl von Ossietzky Universität Oldenburg, Küpkersweg 70, 26129 Oldenburg, Germany \\ ${ }^{2}$ Fraunhofer IWES, Küpkersweg 70, 26129 Oldenburg, Germany
}

Correspondence to: Lukas Vollmer (lukas.vollmer@uni-oldenburg.de)

Received: 9 March 2017 - Discussion started: 6 April 2017

Revised: 19 September 2017 - Accepted: 12 October 2017 - Published: 8 December 2017

\begin{abstract}
The estimation of the cost of energy of offshore wind farms has a high uncertainty, which is partly due to the lacking accuracy of information on wind conditions and wake losses inside of the farm. Wake models that aim to reduce the uncertainty by modeling the wake interaction of turbines for various wind conditions need to be validated with measurement data before they can be considered as a reliable estimator. In this paper a methodology that enables a direct comparison of modeled with measured flow data is evaluated. To create the simulation data, a model chain including a mesoscale model, a large-eddy-simulation (LES) model and a wind turbine model is used. Different setups are compared to assess the capability of the method to reproduce the wind conditions at the hub height of current offshore wind turbines. The 2-day-long simulation of the ambient wind conditions and the wake simulation generally show good agreements with data from a met mast and lidar measurements, respectively. Wind fluctuations due to boundary layer turbulence and synoptic-scale motions are resolved with a lower representation of mesoscale fluctuations. Advanced metrics to describe the wake shape and development are derived from simulations and measurements but a quantitative comparison proves to be difficult due to the scarcity and the low sampling rate of the available measurement data. Due to the implementation of changing synoptic wind conditions in the LES, the methodology could also be beneficial for case studies of wind farm performance or wind farm control.
\end{abstract}

\section{Introduction}

Offshore wind energy still remains an expensive alternative to onshore wind energy, which has been established as one of the cheapest options to generate electricity. One of the reasons for the comparatively high costs of offshore wind energy is the scarcity of long-term atmospheric measurements at existing or planned wind farms. The resource assessment at these locations is difficult and prone to large errors (Walker et al., 2016). In addition, missing measurements during operation prohibit the thorough analysis of turbine malfunctions and unexpected underperformance.

Only a few offshore wind farms deploy permanent met masts that allow for studying the influence of atmospheric conditions on wind farm performance. The available measurements indicate that due to the generally lower level of turbulent kinetic energy offshore compared to onshore, the wakes of the wind turbines are frequently more persistent, which leads to higher wake losses at downwind turbines even over larger distances. An even lower turbulence level caused by stable atmospheric stratification leads to a further increase in wake losses (Barthelmie and Jensen, 2010; Hansen et al., 2012; Dörenkämper et al., 2014).

Several numerical models have been developed with the purpose of calculating the optimal layout of offshore wind farms under consideration of the wake losses. Simplified engineering models allow a fast calculation of multiple wind scenarios and an optimization of wind farm layouts (Sanderse et al., 2011). These steady-state models, however, have a low representation of the flow physics and rely largely on the parametrization of turbulence and on a simplified interaction of turbine and flow.

A high-fidelity solution for wind farm simulations are large-eddy simulations (LESs). Coupled with wind turbine models, LESs provide a detailed solution of the flow inside of a wind farm with a high representation of the relevant 
physics. Due to the high computational costs, LESs of offshore wind farms have been restricted to simulations of idealized atmospheric conditions or to case studies of specific situations, e.g., Churchfield et al. (2012); Dörenkämper et al. (2015); Lu and Porté-Agel (2011). An issue of all wind farm models is the need for validation with measured data to evaluate the capability of the model to reproduce the actual wind conditions and performance of the wind farm under these conditions (Moriarty et al., 2014).

In addition to performance measurements from the data acquisition system of wind turbines, which are often kept confidential, flow measurements using the light detection and ranging methodology (lidar) have become a widespread tool for scientific research. To optimize this technique for model validation, the lidar measurement campaigns have to be designed and postprocessed to allow for a meaningful comparison with simulations. One aspect of the measurement design is the measurement of free flow conditions, which can be used as meteorological boundary conditions for the simulations.

Especially offshore the measurement or derivation of boundary conditions to set up simulations is challenging. For example, onshore LESs are often run with boundary conditions derived from near-surface measurements (e.g., heat flux measurements) and are compared to wind profiles derived from lidar devices (Mirocha et al., 2015; Machefaux et al., 2015) or met masts. This procedure is rarely possible at offshore sites because usually near-surface measurements and wind speed profiles are not available. Furthermore, for many models additional input is required, e.g., the height of the atmospheric boundary layer or a large-scale pressure gradient to drive the flow. These properties have to be estimated or set to default values.

In this paper we investigate a methodology to use profiles and boundary conditions derived from a mesoscale simulation for a continuous LES of an offshore wind turbine wake over several hours. The purpose is to evaluate if this model chain can be used to conduct wake simulations in a wind field with the same turbulent properties and the same profile shape as measured. Measurements from an offshore met mast are used for the evaluation. The model chain is further evaluated by a comparison of the flow distortion by a wind turbine model with the wind field extracted from lidar measurements (van Dooren et al., 2016) of a wake during the simulated time period.

Recently, long-term LESs of multiple days up to 1 year have been run with this approach to study the changes of meteorological conditions at a measurement site (Neggers et al., 2012; Schalkwijk et al., 2015; Heinze et al., 2017). In the context of wind energy, the approach had only been used in Vollmer et al. (2015), in which the measurement and simulation setup of this paper was briefly introduced. Here we extend the study in Vollmer et al. (2015) by analyzing a much larger time interval of measurements and simulations, by a sensitivity study of the method and by a quantitative comparison of wake characteristics.

\section{Data and methodology}

\subsection{Measurement data}

The case study that is analyzed in this paper is based on measurements on 20 February 2014 at the German offshore wind farm Alpha ventus. Two independent data sets were used for comparison with the model results. The simulated ambient wind conditions without turbines were compared to measurements from the met mast FINO1 located at $54^{\circ} 01^{\prime} \mathrm{N}, 6^{\circ} 35^{\prime} \mathrm{E}$. Time series from cup anemometers, wind vanes and temperature probes at different heights as well as sea surface temperature from a buoy were provided by the Bundesamt für Seeschifffahrt und Hydrographie (BSH). These time series provide mean values obtained from averaging over $10 \mathrm{~min}$. For the wind speed, $1 \mathrm{~Hz}$ measurements were made available by DEWI (UL International $\mathrm{GmbH}$ ). The wind directions of the wind vanes at all heights were corrected using a direction-dependent bias (DEWI, personal communication). During the analyzed time period the flow measurement devices did not operate in the mast shadow or in the wake of a wind turbine and should thus provide accurate information about the undisturbed marine atmospheric boundary layer.

The lidar measurements, which were used for comparison with the simulated wakes, are part of a measurement campaign that took place from August 2013 until March 2014. During the analyzed day, two long-range lidar devices (Windcube WLS200S) executed single elevation plan position indicator scans in the wake of turbine AV10 with one lidar positioned on FINO1 and the other one on the converter station of the wind farm (Fig. 1).

The line of sight velocities of the lidars were combined and averaged to get a 10 min mean horizontal vector wind field at hub height (van Dooren et al., 2016). Measurements were filtered at both ends of the range of the carrier-to-noise level to remove low backscatter data as well as reflections from objects. Time periods in which a yaw activity of more than $3^{\circ}$ was observed were removed from the database. Averaging was done on volumes with a diameter of $20 \mathrm{~m}$ centered at hub height. Because both lidars scan over a relatively small range of azimuth angles (Fig. 1), seven (WLS2) and five (WLS3) sweeps over the scan area contribute to the calculation of the mean velocities. The view of the lidar devices to certain areas of the scan is blocked by other wind turbines; thus, a varying total of 100-350 individual line-of-sight wind speed values contribute to the average at each grid point of the final wind field. Grid points with a lower number of values were removed. The coordinate system in which the flow field is presented is oriented north by scanning the distance to the turbines of the wind farm with known geographical coordinate positions. More information on the calculation of 
the vector field from the line-of-sight velocities can be found in van Dooren et al. (2016).

\subsection{Model equations}

Revision 1928 of the Parallelized Large-eddy Simulation Model (PALM) (Maronga et al., 2015) was used for this study with the same numerical schemes as in Vollmer et al. (2016), using a Deardorff model for the sub-grid-scale (SGS) fluxes. The extension of the model equations to include timedependent forcing is based on Heinze et al. (2017) with an extension to include large-scale advection of momentum. The modified equation of motion before applying any approximations is

$$
\begin{aligned}
& \frac{\partial u_{i}}{\partial t}=-\underbrace{u_{j} \frac{\partial u_{i}}{\partial x_{j}}}_{1}-\underbrace{\epsilon_{i j k} f_{j} u_{k}}_{2}-\underbrace{\frac{1}{\rho} \frac{\partial p}{\partial x_{i}}}_{3} \\
& +\underbrace{v_{\mathrm{m}}\left(\frac{\partial^{2} u_{i}}{\partial x_{j}^{2}}+\frac{1}{3} \frac{\partial}{\partial x_{i}} \frac{\partial u_{j}}{\partial x_{j}}\right)}_{4}-\underbrace{\left.\epsilon_{i 3 j} f_{3} u_{\mathrm{g}_{j}}\right|_{\mathrm{LS}}}_{5} \\
& +\underbrace{\left.\frac{\partial u_{i}}{\partial t}\right|_{\mathrm{LS}}}_{6}-\underbrace{\frac{\left\langle u_{i}\right\rangle-u_{i_{\mathrm{LS}}}}{\tau}}_{7},
\end{aligned}
$$

with term 1 representing the momentum advection, term 2 the Coriolis force with the Coriolis parameter $f_{j}$, term 3 the pressure gradient and term 4 the friction terms with the kinematic viscosity of momentum $v_{\mathrm{m}}$. Terms 5-7 are the external forcing terms. For the external forcing, a large-scale velocity denoted by $\left.\right|_{\text {LS }}$ is defined. Term 5 defines a large-scale pressure gradient by prescribing a horizontal geostrophic wind speed $u_{\mathrm{g}}$. Term 6 prescribes a large-scale sink or source of momentum and term 7 is a time relaxation of the momentum towards a large-scale state (Neggers et al., 2012; Heinze et al., 2017).

The momentum relaxation has no physical justification but is used to prevent a drift of the model from the large-scale state. The term depends on the difference between the horizontal average $<u_{i}>$ of each velocity component and the large-scale velocity component $u_{i_{\mathrm{LS}}}$, scaled by a relaxation time constant of $\tau$. All large-scale properties are horizontally homogeneous to preserve the turbulent structures.

The equation for scalars $s \in(\Theta, q)$ is

$$
\frac{\partial s}{\partial t}=-\underbrace{u_{j} \frac{\partial s}{\partial x_{j}}}_{1}+\underbrace{v_{\mathrm{s}} \frac{\partial^{2} s}{\partial x_{j}^{2}}}_{4}+\underbrace{\left.\frac{\partial s}{\partial t}\right|_{\mathrm{LS}}}_{6}-\underbrace{\frac{\langle s\rangle-s_{\mathrm{LS}}}{\tau}}_{7}+\underbrace{S_{\mathrm{s}}}_{8},
$$

with terms 1, 4, 6 and 7 equivalent to the corresponding terms in Eq. (1), with $v_{\mathrm{s}}$ being the molecular diffusivity of the scalar. Term 8 is the surface flux of either the specific humidity $q$ or the potential temperature $\Theta$.

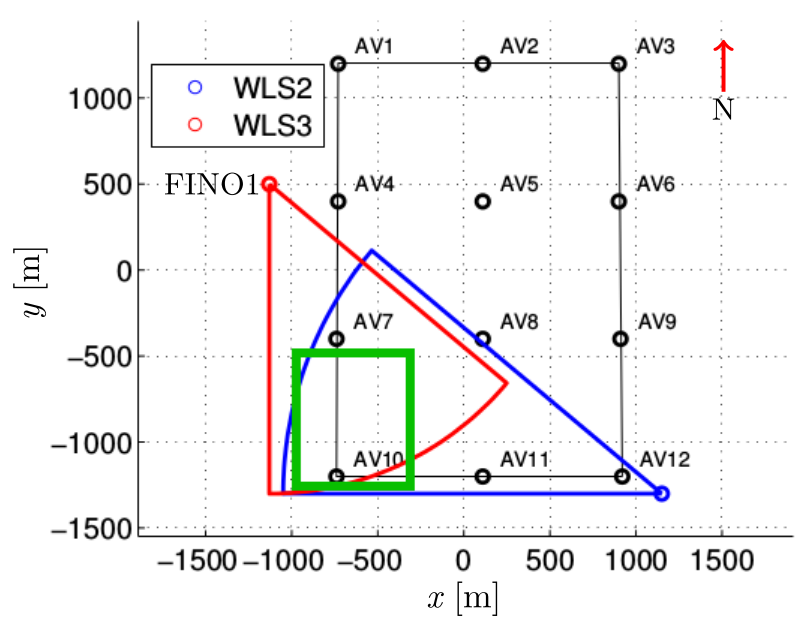

Figure 1. Layout of Alpha ventus and position of the two lidars that were used for the construction of the wind field. Circular segments denote the scan areas of the lidars. The green box denotes the region of the vector wind field reconstruction.

Time dependency of the external forcing is achieved by prescribing profiles of the time-variant geostrophic wind, source terms of horizontal momentum and scalar properties, and of the large-scale state of the relaxation term. The surface fluxes are calculated by making use of the Monin-Obukhov similarity theory, with the values of the surface pressure, temperature and humidity also prescribed by the time-dependent large-scale state.

\section{Simulation of free stream flow}

In this section we analyze the simulation of the ambient conditions with the large-scale forcing derived from the output of a mesoscale simulation. Different parameters are modified to analyze their influence on the results. In Sect. 3.1 we look at the meteorological conditions that were present on the day of the measurements. In Sect. 3.2 we compare profiles from the mesoscale model with the FINO1 measurements and in Sect. 3.3 we compare the LES model output of different setups with the mesoscale model and the FINO1 measurements.

\subsection{Meteorological conditions}

The lidar measurements were conducted on 20 February 2014. After filtering according to the criteria mentioned in Sect. 2.1, $1510 \mathrm{~min}$ time periods remained for further analysis. The 15 time periods can be sorted into three periods, with three measurements starting at 01:00 UTC (night period), nine around 06:00 UTC (morning period) and another three starting at 21:40 UTC (evening period) (Fig. 2).

The wind direction at FINO1 is southwest during the night and south during the rest of the day, with an increase in the wind speed at hub height of the Alpha ventus wind turbines 
(a)

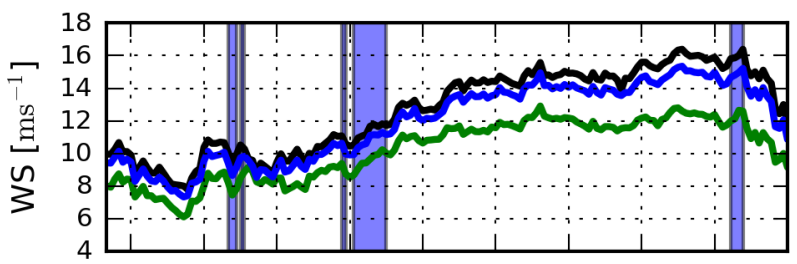

(b)

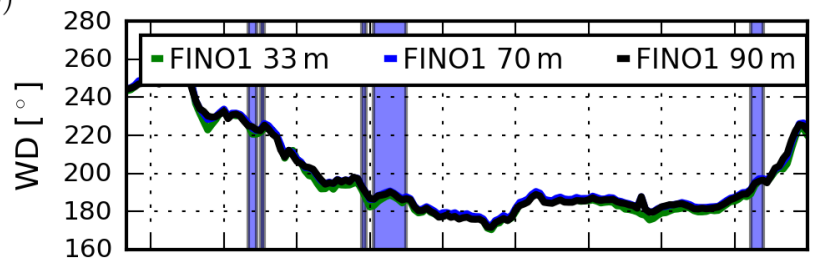

(c)

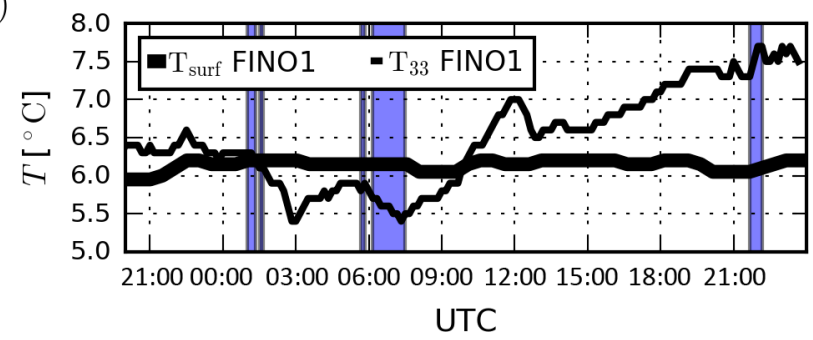

Figure 2. Meteorological conditions on 20 February 2014, as measured at FINO1. (a, b) Wind speed and wind direction at different heights. (c) Temperature as measured at height and the sea surface. The periods of the selected lidar measurements are shaded in blue.

(90 m) from about $8 \mathrm{~ms}^{-1}$ to about $16 \mathrm{~ms}^{-1}$. The day is a rather warm winter day, with the measured temperature at $35 \mathrm{~m}$ ranging from 5.5 to $8^{\circ} \mathrm{C}$. Compared to onshore, the diurnal cycle of surface temperature is very small at offshore locations because of the large heat capacity of the ocean surface. The observed drop of air temperature during the morning hours is thus most likely related to the advection of colder air from the land. The German coast is approximately $45 \mathrm{~km}$ to the south of Alpha ventus. The advected cold air leads to thermally slightly unstable conditions between about 02:00 and 09:00 am. During the rest of the day the stratification is slightly stable.

\subsection{Input data from COSMO-DE}

The profiles for the large-scale tendencies are calculated from the operational analysis of the COSMO-DE model (Baldauf et al., 2009) of the German Weather Service (DWD). COSMO-DE has a horizontal resolution of $2.8 \times 2.8 \mathrm{~km}$ and 50 vertical levels in total, with 20 vertical levels in the lower $3000 \mathrm{~m}$ of the atmospheric boundary layer. The DWD delivers hourly model data.

Following Heinze et al. (2017) three-dimensional and surface data are averaged over a horizontal averaging domain of multiple grid points. The nearest grid cell to the FINO1 coordinates is chosen as the center of the averaging domain.

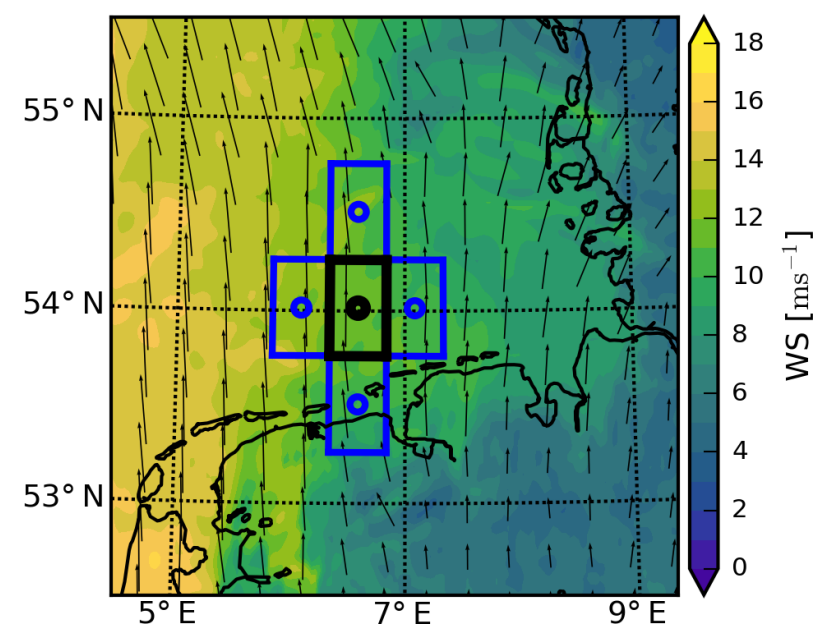

Figure 3. COSMO-DE wind speed and direction on 20 February 2014 at 07:00 UTC on the model level of $73.5 \mathrm{~m}$. The black square marks the averaging domain surrounding FINO1 and the blue squares mark the neighboring domains that are used for the calculation of the gradients.

Because of the necessary spin-up time of the LES for the development of turbulence, $24 \mathrm{~h}$ of simulation time (19 February, whole day) was added. The profile of the geostrophic wind is calculated using the pressure gradient between neighboring averaging domains (Fig. 3). The component of the geostrophic wind along the west-east axis is defined by

$\left.u_{\mathrm{g}_{1}}^{I, J}\right|_{\mathrm{LS}}=-\frac{g f}{\rho^{I, J}} \frac{P^{I, J+1}-P^{I, J-1}}{2 \mathrm{~d} X_{2}}$,

with $P^{I, J}$ and $\rho^{I, J}$ the domain-averaged quantities of density and pressure in domain $(I, J), f$ the Coriolis parameter, and $\mathrm{d} X_{i}$ the length of the averaging domain. The north-south wind component $u_{\mathrm{g}_{2}}^{I, J}$ is defined accordingly. The source terms $\left.\frac{\partial u_{i}}{\partial t}\right|_{\mathrm{LS}},\left.\frac{\partial \Theta}{\partial t}\right|_{\mathrm{LS}}$ and $\left.\frac{\partial q}{\partial t}\right|_{\mathrm{LS}}$ result from the advection into the averaging domain. The source term of the potential temperature $\Theta$ for example is defined by

$$
\begin{aligned}
\left.\frac{\partial \Theta}{\partial t}\right|_{\mathrm{LS}} & =U_{1}^{I, J}\left(\Theta^{I+1, J}-\Theta^{I-1, J}\right) \\
& +U_{2}^{I, J}\left(\Theta^{I, J+1}-\Theta^{I, J-1}\right) .
\end{aligned}
$$

We analyzed the influence of the size of the averaging domain on the profiles required by the LES model by comparing three different quadratic domain sizes with grid lengths of $1 / 8,1 / 2$ and $2^{\circ}$. Figure 4 compares the measured $70 \mathrm{~m}$ wind speed and direction from FINO1 with the horizontal and the geostrophic wind speed and direction from the different averaging domains.

The comparison of the different averaging domains (Fig. 4) shows that the smaller domains contain more fluctuation, but not necessarily at the same time as the measurements. In addition, the geostrophic wind that is calculated 

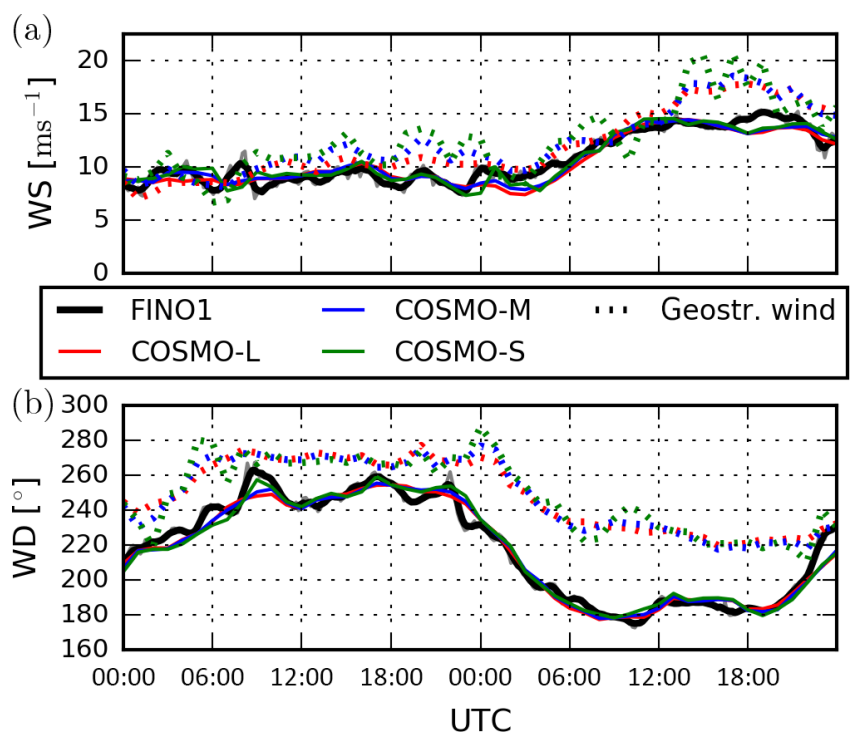

Figure 4. Time series for 19 and 20 February 2014 of (a) wind speed at $70 \mathrm{~m}$. (b) Wind direction at $70 \mathrm{~m}$. FINO1 $1 \mathrm{~h}$ running average (black). COSMO-DE averaging domain sizes of $(1 / 8)^{2 \circ}$ (COSMO-S), $(1 / 2)^{2}$ degrees (COSMO-M) and $2^{2}$ degrees (COSMO-L). Dotted lines represent the calculated geostrophic wind speed and direction at the same height.

from the pressure gradients in the model becomes noisier with decreasing averaging domain size (Fig. 4). Because the geostrophic wind is directly used in the equations of motion, we chose to use the middle-sized domain. It generally contains less noise than the small domain, and in contrast to the large domain, it covers just grid points over the sea, thus representing a horizontally rather homogeneous area.

Figure 5 shows Hovmöller diagrams of most of the largescale forcing data we used for the LES model. As assumed from the measurements, an advection of colder temperature during the morning hours of the second day is visible in the mesoscale simulation (Fig. 5f). The change of wind direction with height is mostly related to the Ekman turning, which can be seen in the differences between the geostrophic and the effective wind direction (Fig. $5 \mathrm{~b}$ and e, respectively).

\subsection{Comparison with met mast data}

To transfer the input profiles from the COSMO-DE time steps and height levels to the LES model, they were linearly interpolated on the vertical LES grid and on the time steps of the simulation. The LESs were initialized with the set of large-scale profiles on 19 February at 00:00 UTC and nudging was applied only after $6 \mathrm{~h}$ to enable a free development of turbulence in the first simulation hours.

All simulations had a domain size of $3200 \mathrm{~m} \times 3200 \mathrm{~m} \times 1700 \mathrm{~m}$ and were run with cyclic boundary conditions. The roughness length of momentum was taken from the COSMO-DE model $\left(z_{0}=1.23 \cdot 10^{-4} \mathrm{~m}\right)$; the roughness lengths of temperature and humidity were $z_{0}^{\Theta, q}=0.1 z_{0}$.

Five different simulations with a rather coarse grid were run with different configurations (Table 1). One of the setups was then run with a finer resolution as basis for the turbine simulations. The chosen setup is regarded as the reference simulation $P_{\text {ref }}$ and the simulation with higher spatial resolution is called $P_{\mathrm{hi}}$. Two alternative relaxation time constants were set in $P_{\tau_{1}}$ and $P_{\tau_{2}}$ and advection of either momentum or potential temperature was switched off in $P_{\partial_{t}} u=0$ and $P_{\partial_{t}} \Theta=0$.

For evaluation we selected the 10 min mean wind speed and direction at $70 \mathrm{~m}$, as they are close to the hub height and also available from the COSMO-DE model. For better comparison the raw $10 \mathrm{~min}$ values from the anemometers and wind vanes were smoothed by means of a $1 \mathrm{~h}$ running mean. The RMSE between each simulation time series and the references is compared in Table 1.

The evaluation shows that switching off momentum advection appears to have the largest influence on the wind speed and wind direction deviation from the input data. Figure $6 \mathrm{a}$ reveals that the impact of momentum advection is largest between 12:00 and 18:00 UTC of the second day, after the increase of the mean wind speed during the morning hours. In this period the mean wind speed remains too high when momentum advection is turned off. As illustrated in Neggers et al. (2012), the flow in the LES contains inertia, which delays the reaction to changing boundary conditions. The nudging term is one option to dampen the inertial fluctuations. Here we find that momentum advection is a complementary option. The advection of potential temperature does not have a large effect on the time series of the wind speed at $70 \mathrm{~m}$. However, Fig. $6 \mathrm{~b}$ shows the importance of the temperature advection for the thermal stability as the sign change of the temperature gradient can indeed be closely related to advection.

The more highly resolved simulation run, which was used as the basis for the turbine simulations, was computed with a relaxation time constant of $\tau=4 \mathrm{~h}$, even though the result with a smaller relaxation time provides a slightly lower deviation from the measurements at hub height. We chose the larger relaxation constant to reduce the influence of the unphysical domain-wide relaxation on the wake simulations. Heinze et al. (2017) and Schalkwijk et al. (2015) found that the overall boundary layer properties are quite independent from the relaxation constant, if the constant is in the magnitude of hours.

The time series of the domain-averaged results of $P_{\mathrm{hi}}$ are compared to the measurements and the large-scale forcing data in Fig. 7. The 10 min turbulence intensity (TI) and the standard deviation of the wind direction are calculated and averaged over multiple virtual met masts evenly distributed over the model domain. The power-law coefficient is calculated for the FINO1 measurements and the LES from a fit to 

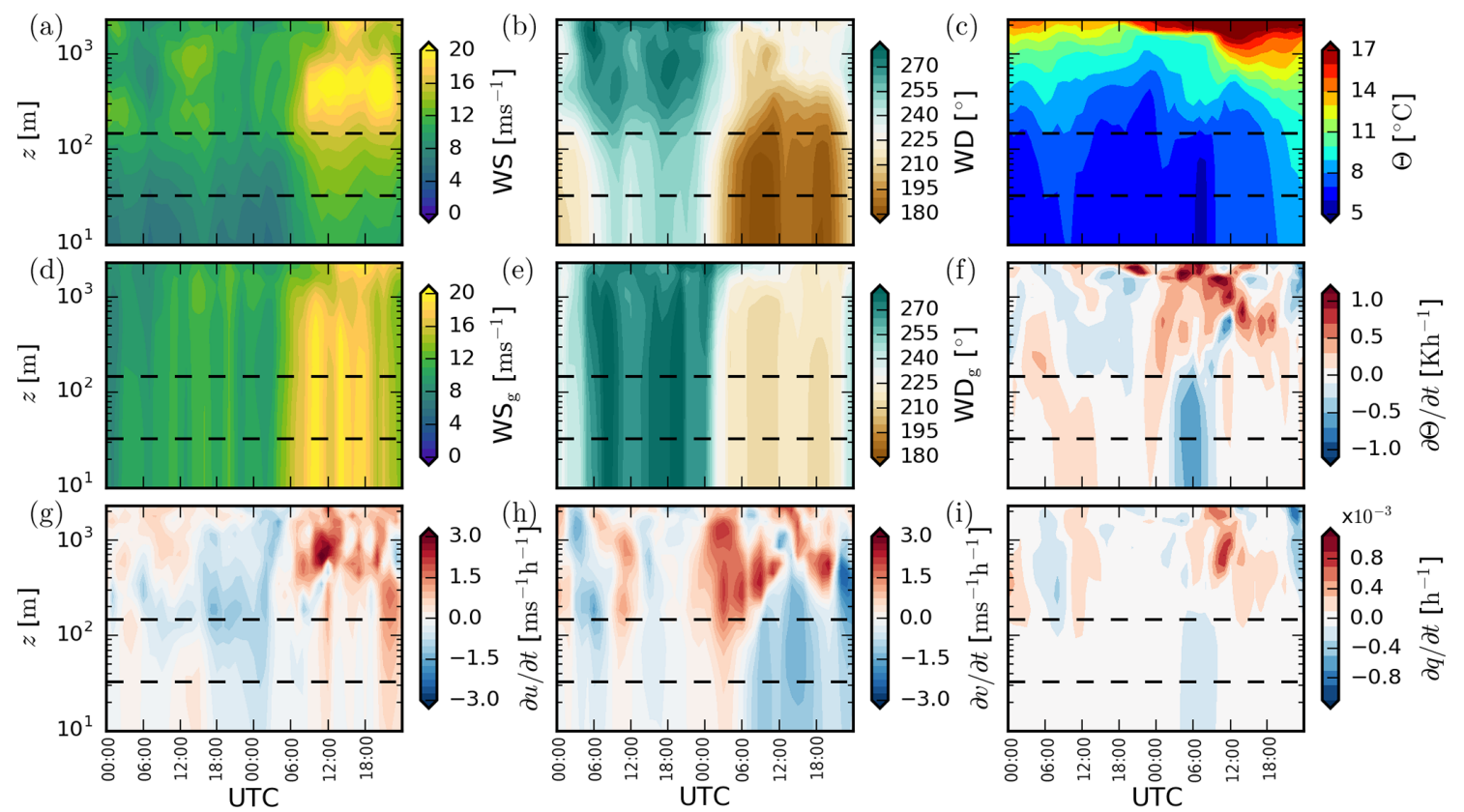

Figure 5. Time development of the vertical input profiles for the LES run. (a) Wind speed, (b) wind direction, (c) potential temperature, (d) geostrophic wind speed, (e) geostrophic wind direction, (f) advection of potential temperature, (g) advection of zonal wind speed, (h) advection of meridional wind speed and (i) advection of humidity. Dashed horizontal lines represent the lower and upper rotor tip heights.

Table 1. Comparison of the different simulation setups and the RMSE of the difference between the time series of simulated wind direction and wind speed of simulations $P_{x}$ and the time series of the COSMO-DE input (C), the FINO1 measurements (F1) and the reference simulation $\left(P_{\text {ref }}\right)$.

\begin{tabular}{lrr|rrrrrr}
\hline & \multicolumn{2}{c|}{ Setup } & \multicolumn{6}{c}{ RMSE } \\
\cline { 2 - 8 } $\operatorname{Sim}$ & $\begin{array}{r}\tau \\
{[\mathrm{h}]}\end{array}$ & $\begin{array}{r}\Delta x, z \\
{[\mathrm{~m}]}\end{array}$ & $\begin{array}{r}\mathrm{WD}_{\mathrm{C}} \\
{\left[{ }^{\circ}\right]}\end{array}$ & $\begin{array}{r}\mathrm{WD}_{\mathrm{F} 1} \\
{\left[{ }^{\circ}\right]}\end{array}$ & $\begin{array}{r}\mathrm{WD}_{P_{\text {ref }}} \\
{\left[{ }^{\circ}\right]}\end{array}$ & $\begin{array}{r}\mathrm{WS}_{\mathrm{C}} \\
{\left[\mathrm{ms}^{-1}\right]}\end{array}$ & $\begin{array}{r}\mathrm{WS}_{\mathrm{FINO} 1} \\
{\left[\mathrm{~ms}^{-1}\right]}\end{array}$ & $\begin{array}{r}\mathrm{WS}_{P_{\text {ref }}} \\
{\left[\mathrm{ms}^{-1}\right]}\end{array}$ \\
\hline$P_{\text {ref }}$ & 4 & $20 / 10$ & 5.8 & 6.6 & - & 0.79 & 1.08 & - \\
$P_{\tau_{1}}$ & 1.5 & $20 / 10$ & 4.0 & 5.1 & 2.5 & 0.73 & 0.99 & 0.16 \\
$P_{\tau_{2}}$ & 48 & $20 / 10$ & 7.5 & 8.3 & 2.8 & 0.70 & 1.10 & 0.36 \\
$P_{\partial_{t} u=0} u$ & 4 & $20 / 10$ & 8.5 & 9.4 & 6.2 & 1.53 & 1.76 & 1.02 \\
$P_{\partial_{t}} \Theta=0$ & 4 & $20 / 10$ & 6.9 & 7.6 & 1.8 & 0.77 & 1.08 & 0.14 \\
$P_{\mathrm{hi}}$ & 4 & $5 / 5$ & 5.7 & 6.5 & 1.4 & 0.46 & 0.91 & 0.42 \\
\hline
\end{tabular}

the data between 33 and $90 \mathrm{~m}$, and for COSMO-DE by using the model levels at 35 and $73 \mathrm{~m}$.

LES wind speed and wind direction follow the general trend of the input and measurement data. The averaged magnitude of the turbulent fluctuations on the $10 \mathrm{~min}$ scale is also reproduced. The largest discrepancy between simulation and measurements exists in the shear of the vertical wind profile which is almost constantly lower in the LES.

The destabilization of the boundary layer is observable as a decrease of the vertical shear of the LES and the measurements (Fig. 7e) on the second day between 00:00 and 06:00 UTC. The event appears to occur earlier in reality than in the simulations, which is likely related to the earlier change of sign of the temperature gradient (Fig. 6). The re- stratification also starts later in the LES and the vertical shear remains constantly lower during the rest of the day.

The comparison of modeled and measured time series shows that the measurements contain additional fluctuations that are not replicated by the model chain of mesoscale and microscale models. Figure 8 compares the power spectra of the two time series at hub height. The LES data show the typically stronger drop in the highest frequencies related to the cutoff by the implicit SGS filtering. At longer periods of about 0.5 to $12 \mathrm{~h}$, the gradient of the measured energy cascade is maintained, while the simulation contains less energy in this range. The model chain thus enables the replication of the synoptic-scale motions and the boundary layer turbulence but fails to reproduce the impact of mesoscale motions. 

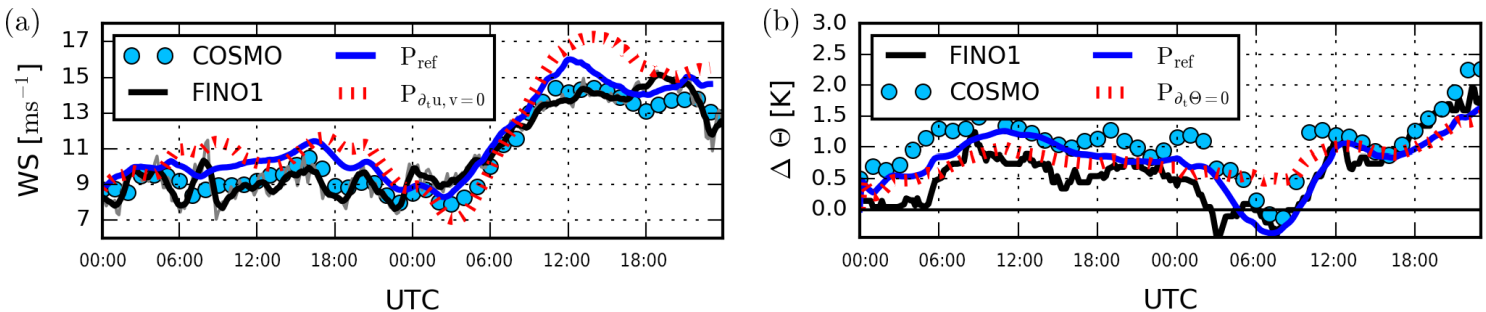

Figure 6. (a) Wind speed at $70 \mathrm{~m}$ with and without momentum advection. (b) Potential temperature difference between $35 \mathrm{~m}$ and the surface with and without temperature advection.
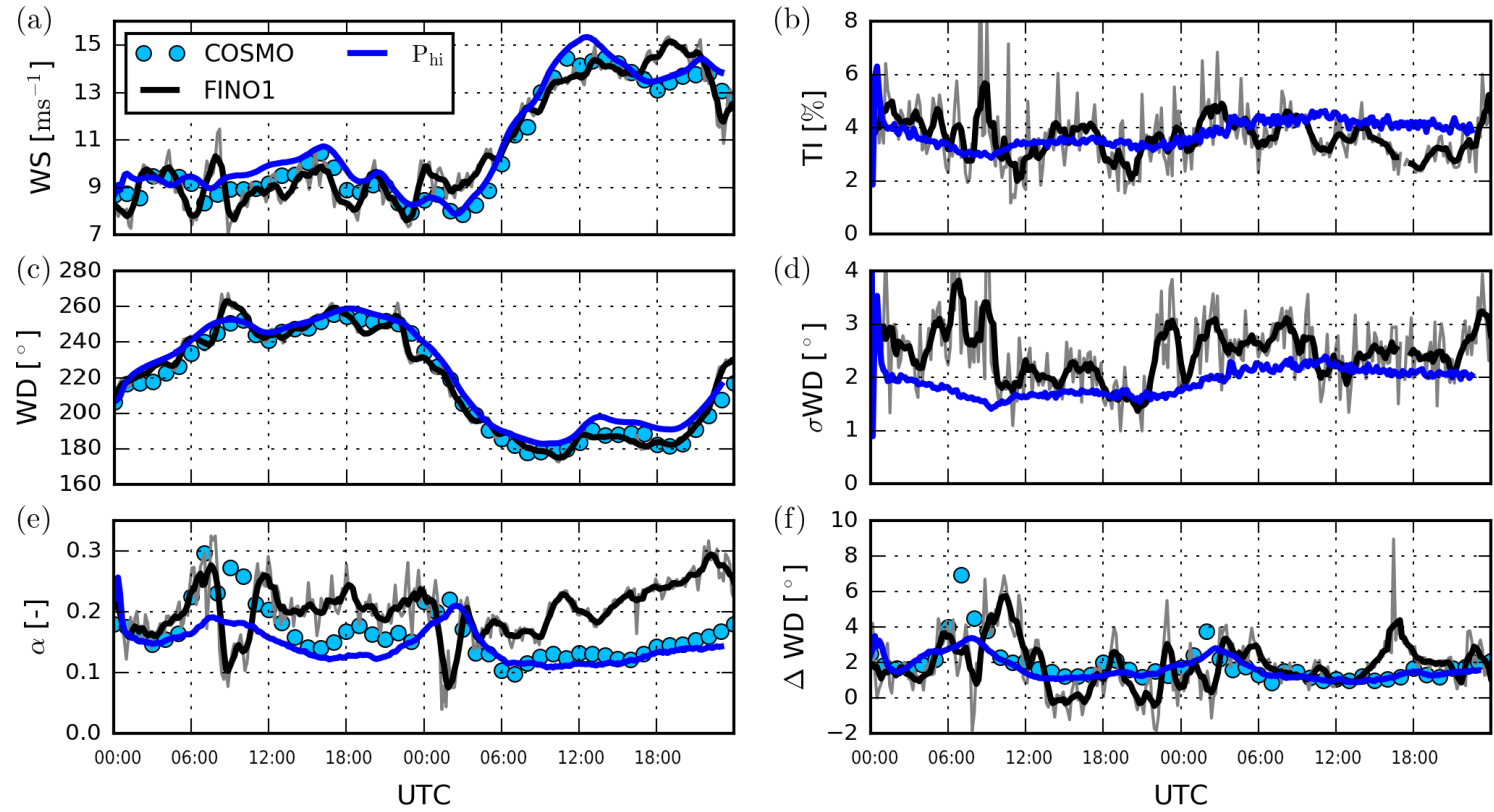

Figure 7. Comparison of $P_{\mathrm{hi}}$ to COSMO-DE and $1 \mathrm{~h}$ running means (black) and $10 \mathrm{~min}$ averages (grey) of FINO1 measurements. All time series are at $70 \mathrm{~m}$, if not otherwise specified. (a) Wind speed, (b) turbulence intensity, (c) wind direction, (d) 10 min standard deviation of the wind direction, (e) vertical power-law coefficient and (f) change of the wind direction between 33 and $70 \mathrm{~m}$.

The horizontal averaging of the mesoscale model output to derive smooth boundary conditions might factor into the result. Vincent et al. (2013), however, show that even current highly resolved mesoscale model output is not able to capture mesoscale fluctuations. As this paper only considers a small time period, we refer to Schalkwijk et al. (2015) for a more complete discussion of this topic.

\section{Wind turbine wake simulations}

\subsection{Model setup}

The wind turbine wake simulations are run with the same domain and setup as the high-resolution simulation $P_{\mathrm{hi}}$, just with the added body forces from the wind turbine, placed in the center of the domain. Due to the cyclic horizontal boundary conditions, the wind turbine wake reenters the domain through the southern boundary after having left it through the northern boundary. However, as the wind direction in the

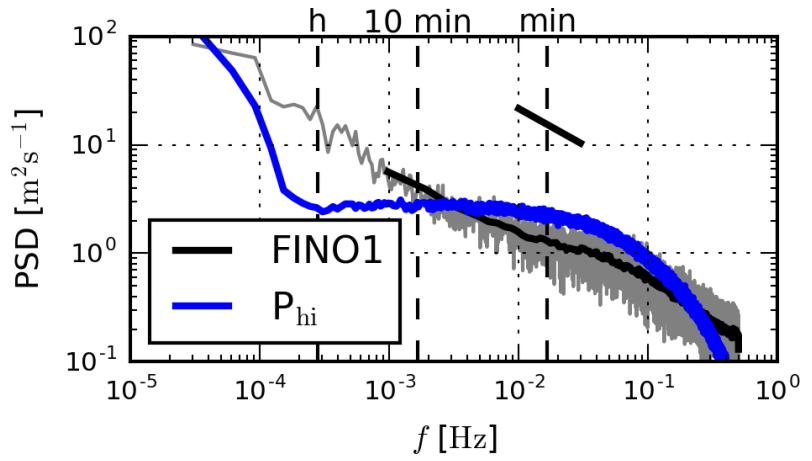

Figure 8. Power spectral density derived from the $1 \mathrm{~Hz}$ measurements at $90 \mathrm{~m}$ height and the simulation time series at $90 \mathrm{~m}$. Grey and black lines represent different window sizes for the Fourier transformation. The short black lines denote the slope of the Kolmogorov cascade. 
(a)

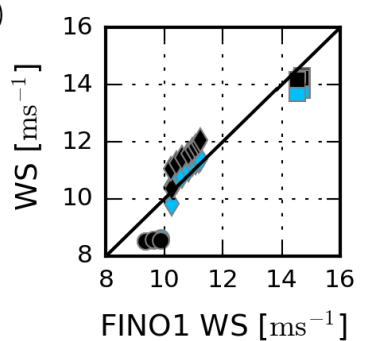

(c)

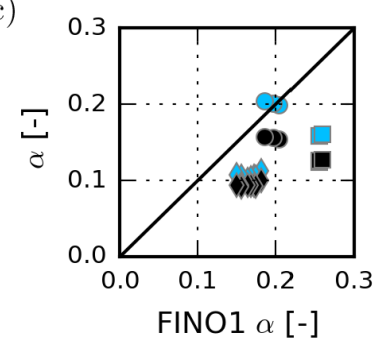

(e)

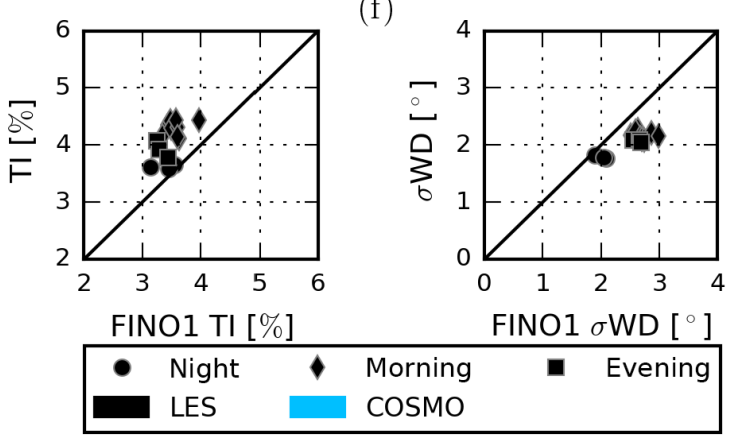

Figure 9. Comparison of the simulated state of the boundary layer with the measured state during the $1510 \mathrm{~min}$ time periods of the lidar measurements. The night period is between 01:00 and 01:40 UTC, the morning period between 05:40 and 07:30 UTC, and the evening period between 21:40 and 22:10 UTC.

simulations is never directly from the south, the turbine is not subject to its own wake. The turbulence of the wake still modifies the state of the atmospheric boundary layer after some time. Thus, we simulated only intervals of $30 \mathrm{~min}$ with the wind turbine, preceded by a $3 \mathrm{~min}$ precursor phase for the development of the wake. Wind fields from $P_{\text {hi }}$ were used as the initial fields of these simulations.

An enhanced actuator disc model with rotation (ADM-R) is used to calculate the forces of the wind turbine on the flow (Witha et al., 2014). The model divides the rotor surface into annulus segments, and the local velocities at the segments and tabulated lift and drag coefficients are used to calculate lift and drag forces. The tower and nacelle of the turbine are parameterized by constant drag coefficients. The parameterized wind turbine AV10 is an Adwen AD 5116 with a rotor diameter $(D)$ of $116 \mathrm{~m}$ and a rated power of $5 \mathrm{MW}$. The hub height of the turbine is at $90 \mathrm{~m}$. Adaptation to the current wind conditions is ensured by a baseline gen- erator torque and pitch controller as described in Jonkman et al. (2009) and a simple yaw controller. Simulations of the generator torque and pitch controller in idealized conditions were performed to ensure that the reference thrust and power curves are replicated. The yaw controller is implemented as described in Storey et al. (2013), with a temporal averaging window of the wind direction of $30 \mathrm{~s}$ and a tolerated maximum misalignment of $5^{\circ}$.

\subsection{Comparison with lidar measurements}

Figure 9 compares parameters that indicate the state of the atmospheric boundary layer measured at FINO1 with the simulated state during the selected 1510 min time periods of the lidar measurements at Alpha ventus. As discussed earlier, the biggest disagreement is found in the vertical shear, which is constantly lower in the simulations. The TI is slightly higher in the simulations. Changes of atmospheric stability are small between the different times of measurements with the night and evening periods in slightly stable conditions and the morning period in neutral conditions according to the classification in Peña et al. (2010), with the Monin-Obukhov length derived from the model fluxes.

For the wind turbine the three periods represent different operating conditions. With a rated wind speed of the turbine of $12.5 \mathrm{~ms}^{-1}$, the wind speed range lies below rated wind speed during the night period. Below rated wind speed the turbine's power and thrust coefficient are nearly constant. During the evening period the wind speed is clearly above rated conditions; thus, the rotor speed is controlled by collective pitch movement of the blades, and the thrust coefficient decreases with increasing wind speed. The morning period represents conditions that are around rated wind speed at which the thrust coefficient should be lower and pitch control is occasionally applied.

Figure 10 shows $10 \mathrm{~min}$ averages of the normalized hubheight wind speed during selected time intervals from simulation and measurements. For better comparison, the LES results were averaged on cubes with a side length of $20 \mathrm{~m}$ centered at hub height, similar to the postprocessing of the lidar data as explained in Sect. 2.1. The slight disagreement of the inflow wind speed was approached by normalizing the velocities of both flow fields. Lidar and LES wind speeds are normalized with the $90 \mathrm{~m}$ wind speed measured in the non-wake measurements or simulation data, respectively. To further remove the disagreement caused by the difference in wind direction, the flow fields were rotated in Fig. 10 (rightmost panels), so that the wake propagates along the $y$ axis.

The 10 min averaging does not apparently filter all turbulent structures of the measured wake field. This is most probably due to the much lower sampling rate of the lidar measurements. Approximately 8000 single values contribute to the average on the $20 \mathrm{~m}$ grid in the LES, considering a time step of about $2 \mathrm{~Hz}$ and the original grid resolution of $5 \mathrm{~m}$. In contrast, the sample velocities contributing to the lidar aver- 

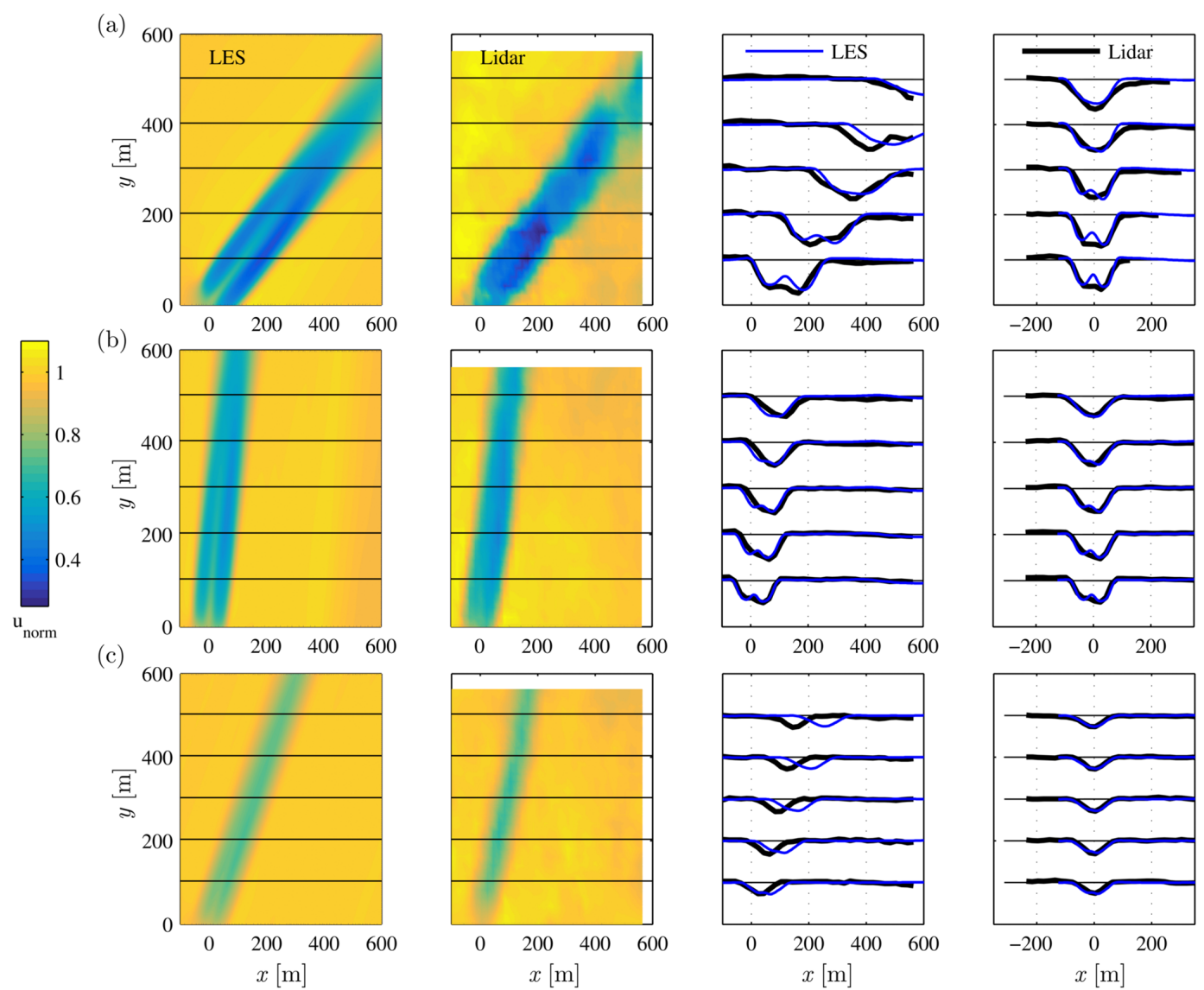

Figure 10. Normalized wind fields from LES and lidar measurements. The third column shows horizontal cross sections along the lines at constant $y$, as depicted in the first two columns. The fourth column shows cross sections at the same distances with the zero coordinate coinciding with the center line of the wake. The full vertical distance between the horizontal lines in the cross section panels equals a normalized velocity of one. The rows represent individual 10 min time periods starting at (a) 01:30, (b) 06:50 and (c) 21:40 UTC.
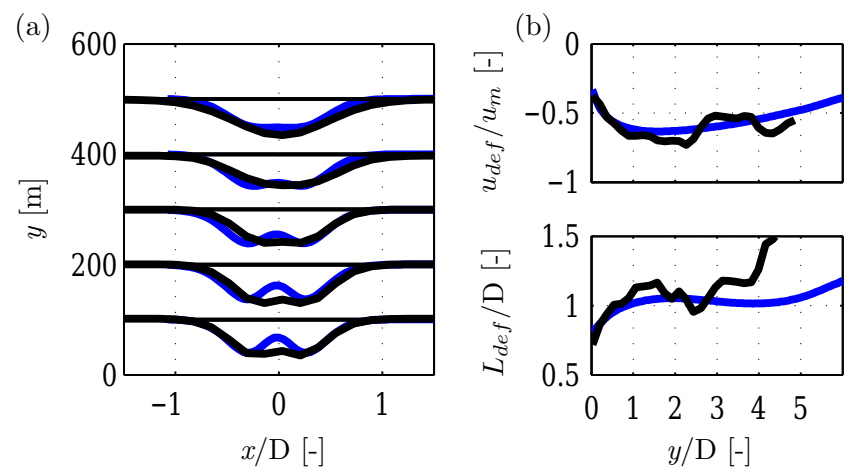

Figure 11. Fitted functions to the wake at 01:30 UTC. (a) Profiles in different distances. (b) Development of normalized deficit and wake width. LES (blue) and lidar (black).

age vary from 100 to 350 individual line-of-sight wind speed values and are not evenly distributed in space and time.

The results show that the unrotated wakes (Fig. 10, third column) match very well during the morning period where
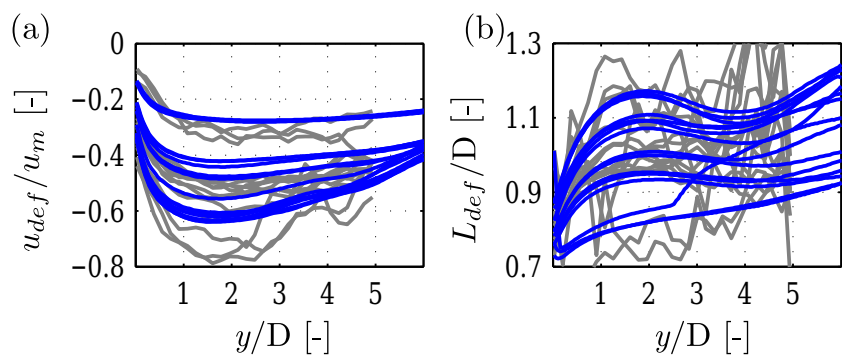

Figure 12. (a) Wake deficit and (b) wake width development from LES (blue) and lidar (grey).

the wind direction appears to be nearly identical. In this period an asymmetry in the horizontal profile of the wake is also clearly visible, a phenomena related to the interaction of vertical wind shear with the rotation of the wake, as shown for example in Vollmer et al. (2015). The amplitude of the wake deficit appears to be best simulated in the evening period when the turbine operates above rated power. The lower 
(a)

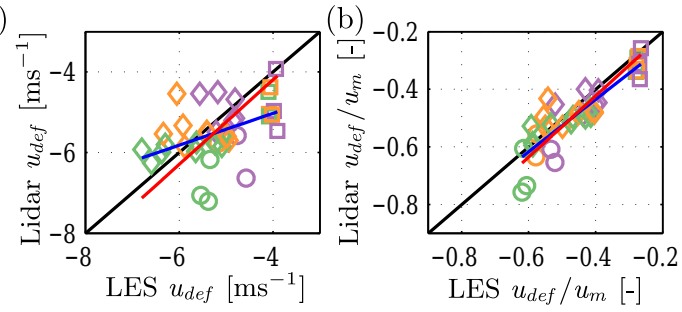

(c)

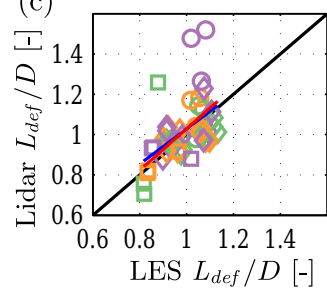

(d)

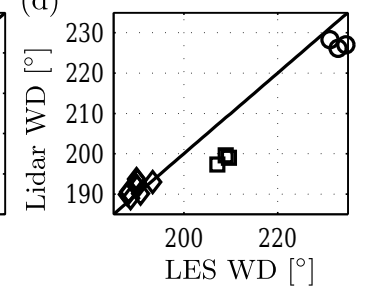

$\mathrm{y}=300 \mathrm{~m}$

- $\mathrm{y}=400 \mathrm{~m}$

$\mathrm{y}=500 \mathrm{~m}$

O Night

$\diamond$ Morning

$\square$ Evening

Figure 13. Scatter plots of the properties derived from the Gaussian-like fit to the wake profiles of (a) the wake deficits, (b) the normalized deficits, (c) the wake widths and (d) the wind direction derived from the propagation direction of the wakes. The three different time intervals are marked with different markers as in Fig. 9. Colors represent the downstream distance from the turbine. Lines show simple linear regression (blue) and regression through the origin (red).

thrust leads to a wake that shows no signs of double minimums in the near wake as visible in the other measurements and simulations.

In the following an attempt is made to make a quantitative comparison between the measured and simulated wakes. To derive statistics of the wakes, the profiles of all 15 time intervals were fitted to a curve consisting of two Gaussian-like functions:

$u_{w}(x)=b \exp \left(-\left(\frac{x-c}{d}\right)^{2}\right)-e b \exp \left(-\left(\frac{x-c}{f d}\right)^{2}\right)$.

Within this function, the central region of lower deficit in the near wake is represented by the second exponential function, with $e, f \in[0,1]$. The wake width (90th percentile) was defined as $L_{\mathrm{def}}=\sqrt{2} \cdot 1.64 \cdot d$, as deficit $u_{\mathrm{def}}$ the minimum value of $u_{w}$ was chosen. Figure 11 shows an example of the fits to the data and Fig. 12 shows the fitted deficit and wake width for all time periods. Minimums of the deficits can be identified in both data sets at a distance of between 1 and $2 \mathrm{D}$ downstream for the high-thrust situations and at a distance of about $3 \mathrm{D}$ for the low-thrust situations. In general the trajectories from the measurements exhibit much more noise, which makes a comparison especially of the wake widths difficult.

A direct comparison of the wake properties at selected downstream distances is presented in Fig. 13. Regression through the origin shows quite a good agreement between lidar and LES wake deficit, though the LES shows a tendency to simulate a higher wake deficit during the morning period and lower deficits otherwise. As no time series of thrust measurements of the turbine were available, we can only speculate if the difference is related to a different thrust of the turbine than in the constructors' specification or if either model or measurement data are inaccurate. The spread decreases by normalizing the deficits and the slope of regression between simulated and measured normalized deficits is 1.05 . The simulated wakes are slightly wider on average, except when the turbine is being operated in below-rated conditions (night period), in which the measured wakes are both wider and have a higher deficit.

\section{Discussion}

As demonstrated in this paper, the forcing of LES with mesoscale-model profiles allows for time-dependent LESs that change according to the synoptic meteorological conditions. Thus, a transient state of the atmospheric boundary layer can be used for the analysis of wind farm model performance. This allows, for example, for a direct validation of wake simulations with measured data, which represents a different approach from the classical statistically derived validation data for wind farm models.

The comparison of the ambient flow created by the model chain with met mast data indicates that the synoptic trend of wind speed and direction is maintained and that the average properties of the simulated wind profile during the 2-day period are close to the properties of the measured wind profile. Time series and power spectra, however, reveal a gap of energy contained in the mesoscale fluctuation range. These fluctuations might be partly resolved with a much larger LES domain, if they originate from thermal effects (Schalkwijk et al., 2015). An inclusion of measurement data as a relaxation data set (Rodrigo et al., 2016) might also lead to a closer tracking of the measurements but requires measurements at higher heights than available in this case.

As this paper only looks at a very short time period, we refrain from drawing general conclusions about the model chain's ability to replicate the evolving state of the atmospheric boundary layer and refer to Schalkwijk et al. (2015) and Heinze et al. (2017), who analyze longer time periods. For wind energy purposes we think that the mesoscale model remains the crucial part of the presented model chain to improve the spectral and vertical representation of the wind field. However, at least for the spectral part, current combinations of models and reanalysis data do not appear to be sufficient (Vincent et al., 2013).

The comparison of the wake simulations with the measured wakes represents one of the suggested applications of the model chain. Instead of averaging over similar wind profile states, a direct time series comparison is performed. The visual comparison of simulated with measured wakes shows a good match, indicating that wind direction and wake pro- 
file are well replicated. Derived wake statistics of the downstream development, however, reveal that the available measurement data still require a more statistical treatment to be able to draw a conclusion about the goodness of the wake representation in the simulations.

Alternatives to the presented approach for LES of wind turbines in the atmospheric boundary layer are idealized quasi-stationary setups or nested LESs inside a mesoscale model. Both of these approaches have advantages and disadvantages compared to the method in this paper. An idealized setup enables the study of an identified quasi-stationary state of the atmospheric boundary layer in detail, as in Vollmer et al. (2016) and Mirocha et al. (2015), for example, or the replication of an idealized transient state (Abkar et al., 2016). For the simulation of observed transient states, however, information from a larger-scale model becomes necessary. Boundary conditions of quasi-stationary states can also be derived from a mesoscale model with the method presented in this paper, if no sufficient measurement information is available.

The approach of a nested LES domain inside of a mesoscale-model domain might enable the inclusion of frequencies of the flow in the range of mesoscale fluctuations or the advection of a different level of turbulence created by upstream obstacles. However, it needs a large LES domain for the development of microscale turbulence (MuñozEsparza et al., 2014) or a good solution to initialize the turbulence at the inflow boundary. It also requires running a mesoscale model in parallel, which is not necessary for the offline coupling approach, which can be started from external data sources by the use of the COSMO-DE data, as shown in this paper.

\section{Conclusions}

In this paper we introduce and test a method to simulate a wind turbine wake in the offshore atmospheric boundary layer with LESs driven by forcing derived from a mesoscale simulation. The methodology enables the simulation of a transient state of the atmospheric boundary layer for the evaluation of wind farm performance or the validation of wake simulations. The comparison with met mast data shows that the model chain is able to reproduce the synoptic trends and the boundary layer turbulence of the marine wind field during the 2 days analyzed. Most of the mesoscale fluctuations found in the measurements are not replicated, which is most likely related to the deficiencies of the mesoscale model. The wake simulations are compared to lidar measurements downstream of an Alpha ventus turbine. In certain periods the modeled and measured wakes are very similar, as especially the wind direction matches well. A direct comparison of measures to describe the downstream wake development proves to be difficult with a high scatter of the measured wakes. Thus, the limited data set of the lidar mea- surements and the still-prevailing turbulent structures in the 10 min averages of the data make it difficult to validate the performance of the whole model chain. We think that the methodology might be especially valuable for transient nonneutral states of the atmospheric boundary layer, in which the boundary conditions to set up LESs are difficult to derive. In these cases, the method presented might not only be valuable for the comparison of simulations with measurement data but could also be applied to study wind turbine or wind farm control in changing wind conditions.

Data availability. The simulation data and the model implementation code could be made available in the framework of a cooperation agreement. Please contact the corresponding author for further information.

Competing interests. The authors declare that they have no conflict of interest.

Acknowledgements. The authors gratefully acknowledge the efforts of the Wind Energy Systems group of ForWind who carried out the lidar measurements at Alpha ventus, including Jörge Schneemann, Davide Trabucchi, Juan-Jose Trujillo and Stephan Voß. The work presented in this study was conducted within the German national research projects "GWWakes" and "OWEA Loads" (FKZ 0325397A and 0325577B), funded by the Federal Ministry for Economic Affairs and Energy (BMWi) and within the project "ventus efficiens" (ZN3024, Ministry for Science and Culture of Lower Saxony). Computer resources have been partly provided by the North-German Supercomputing Alliance (HLRN). We thank the Deutscher Wetterdienst (DWD) for providing analysis data. We thank the BSH and DEWI for providing measurement data from FINO1 (FINO project by BMWi).

Edited by: Luciano Castillo

Reviewed by: two anonymous referees

\section{References}

Abkar, M., Sharifi, A., and Porté-Agel, F.: Wake flow in a wind farm during a diurnal cycle, J. Turbul., 17, 420-441, https://doi.org/10.1080/14685248.2015.1127379, 2016.

Baldauf, M., Förstner, J., Klink, S., Reinhardt, T., Schraff, C., Seifert, A., and Stephan, K.: Kurze Beschreibung des LokalModells Kürzestfrist COSMO-DE (LMK) und seiner Datenbanken auf dem Datenserver des DWD, Deutscher Wetterdienst, Geschäftsbereich Forschung und Entwicklung, Offenbach, Germany, 2009.

Barthelmie, R. J. and Jensen, L. E.: Evaluation of wind farm efficiency and wind turbine wakes at the Nysted offshore wind farm, Wind Energy, 13, 573-586, https://doi.org/10.1002/we.408, 2010.

Churchfield, M. J., Lee, S., Michalakes, J., and Moriarty, P. J.: A numerical study of the effects of atmospheric and wake turbu- 
lence on wind turbine aerodynamics, J. Turbulence, 13, 1-32, https://doi.org/10.1080/14685248.2012.668191, 2012.

Dörenkämper, M., Tambke, J., Steinfeld, G., Heinemann, D., and Kühn, M.: Atmospheric Impacts on Power Curves of MultiMegawatt Offshore Wind Turbines, J. Phys. Conf. Ser., 555, 012029, https://doi.org/10.1088/1742-6596/555/1/012029, 2014.

Dörenkämper, M., Witha, B., Steinfeld, G., Heinemann, D., and Kühn, M.: The impact of stable atmospheric boundary layers on wind-turbine wakes within offshore wind farms, J. Wind Eng. Ind. Aerod., 144, 146-153, https://doi.org/10.1016/j.jweia.2014.12.011, 2015.

Hansen, K. S., Barthelmie, R. J., Jensen, L. E., and Sommer, A.: The impact of turbulence intensity and atmospheric stability on power deficits due to wind turbine wakes at Horns Rev wind farm, Wind Energy, 15, 183-196, https://doi.org/10.1002/we.512, 2012.

Heinze, R., Moseley, C., Böske, L. N., Muppa, S. K., Maurer, V., Raasch, S., and Stevens, B.: Evaluation of large-eddy simulations forced with mesoscale model output for a multi-week period during a measurement campaign, Atmos. Chem. Phys., 17, 7083-7109, https://doi.org/10.5194/acp-17-7083-2017, 2017.

Jonkman, J. M., Butterfield, S., Musial, W., and Scott, G.: Definition of a 5-MW reference wind turbine for offshore system development, Technical Report NREL/TP-500-38060, National Renewable Energy Laboratory, 1617 Cole Boulevard, Golden, Colorado 80401-3393, https://doi.org/10.2172/947422, 2009.

Lu, H. and Porté-Agel, F.: Large-eddy simulation of a very large wind farm in a stable atmospheric boundary layer, Phys. Fluids, 23, 065101, https://doi.org/10.1063/1.3589857, 2011.

Machefaux, E., Larsen, G. C., Koblitz, T., Troldborg, N., Kelly, M. C., Chougule, A., Hansen, K. S., and Rodrigo, J. S.: An experimental and numerical study of the atmospheric stability impact on wind turbine wakes, Wind Energy, 19, 1785-1805, https://doi.org/10.1002/we.1950, 2015.

Maronga, B., Gryschka, M., Heinze, R., Hoffmann, F., KananiSühring, F., Keck, M., Ketelsen, K., Letzel, M. O., Sühring, M., and Raasch, S.: The Parallelized Large-Eddy Simulation Model (PALM) version 4.0 for atmospheric and oceanic flows: model formulation, recent developments, and future perspectives, Geosci. Model Dev., 8, 2515-2551, https://doi.org/10.5194/gmd8-2515-2015, 2015.

Mirocha, J. D., Rajewski, D. A., Marjanovic, N., Lundquist, J. K., Kosovic, B., Draxl, C., and Churchfield, M. J.: Investigating wind turbine impacts on near-wake flow using profiling lidar data and large-eddy simulations with an actuator disk model, J. Renew. Sustain. Ener., 7, 043143, https://doi.org/10.1063/1.4928873, 2015.

Moriarty, P., Rodrigo, J. S., Gancarski, P., Chuchfield, M., Naughton, J. W., Hansen, K. S., Machefaux, E., Maguire, E., Castellani, F., Terzi, L., Breton, S.-P., and Ueda, Y.: IEA-Task 31 WAKEBENCH: Towards a protocol for wind farm flow model evaluation, Part 2: Wind farm wake models, J. Phys. Conf. Ser., 524, 012185, https://doi.org/10.1088/1742-6596/524/1/012185, 2014.

Muñoz-Esparza, D., Kosovic, B., Mirocha, J., and van Beeck, J.: Bridging the Transition from Mesoscale to Microscale Turbulence in Numerical Weather Prediction Models, Bound.-Lay. Meteorol., 153, 409-440, https://doi.org/10.1007/s10546-0149956-9, 2014.
Neggers, R. A. J., Siebesma, A. P., and Heus, T.: Continuous Single-Column Model Evaluation at a Permanent Meteorological Supersite, B. Am. Meteorol. Soc., 93, 1389-1400, https://doi.org/10.1175/BAMS-D-11-00162.1, 2012.

Peña, A., Gryning, S.-E., and Mann, J.: On the length-scale of the wind profile, Q. J. Roy. Meteorol. Soc., 136, 2119-2131, https://doi.org/10.1002/qj.714, 2010.

Rodrigo, J. S., Churchfield, M., and Kosovic, B.: A wind energy benchmark for ABL modelling of a diurnal cycle with a nocturnal low-level jet: GABLS3 revisited, J. Phys. Conf. Ser., 753, 032024, https://doi.org/10.1088/1742-6596/753/3/032024, 2016.

Sanderse, B., van der Pijl, S., and Koren, B.: Review of computational fluid dynamics for wind turbine wake aerodynamics, Wind Energy, 14, 799-819, https://doi.org/10.1002/we.458, 2011.

Schalkwijk, J., Jonker, H. J. J., Siebesma, A. P., and Bosveld, F. C.: A Year-Long Large-Eddy Simulation of the Weather over Cabauw: An Overview, Mon. Weather Rev., 143, 828-844, https://doi.org/10.1175/MWR-D-14-00293.1, 2015.

Storey, R., Norris, S., and Cater, J.: Large Eddy Simulation of Wind Events Propagating through an Array of Wind Turbines, in: Proceedings of the World Congress on Engineering 2013 Vol III, WCE 2013, 3-5 July 2013, London, UK, 2013.

van Dooren, M. F., Trabucchi, D., and Kühn, M.: A Methodology for the Reconstruction of 2D Horizontal Wind Fields of Wind Turbine Wakes Based on Dual-Doppler Lidar Measurements, Remote Sens., 8, 809-823, https://doi.org/10.3390/rs8100809, 2016.

Vincent, C., Larsén, X., Larsen, S., and Sørensen, P.: Cross-Spectra Over the Sea from Observations and Mesoscale Modelling, Bound.-Lay. Meteorol., 2, 297-318, https://doi.org/10.1002/joc.2175, 2013.

Vollmer, L., van Dooren, M., Trabucchi, D., Schneemann, J., Steinfeld, G., Witha, B., Trujillo, J., and Kühn, M.: First comparison of LES of an offshore wind turbine wake with dualDoppler lidar measurements in a German offshore wind farm, J. Phys. Conf. Ser., 625, 012001, https://doi.org/10.1088/17426596/625/1/012001, 2015.

Vollmer, L., Steinfeld, G., Heinemann, D., and Kühn, M.: Estimating the wake deflection downstream of a wind turbine in different atmospheric stabilities: an LES study, Wind Energ. Sci., 1, 129141, https://doi.org/10.5194/wes-1-129-2016, 2016.

Walker, K., Adams, N., Gribben, B., Gellatly, B., Nygaard, N. G., Henderson, A., Marchante Jimémez, M., Schmidt, S. R., Rodriguez Ruiz, J., Paredes, D., Harrington, G., Connell, N., Peronne, O., Cordoba, M., Housley, P., Cussons, R., Håkansson, M., Knauer, A., and Maguire, E.: An evaluation of the predictive accuracy of wake effects models for offshore wind farms, Wind Energy, 19, 979-996, https://doi.org/10.1002/we.1871, 2016.

Witha, B., Steinfeld, G., Dörenkämper, M., and Heinemann, D.: Large-eddy simulation of multiple wakes in offshore wind farms, J. Phys. Conf. Ser., 555, 012108, https://doi.org/10.1088/17426596/555/1/012108, 2014. 